\title{
Polycystic Ovarian Syndrome and Narcolepsy: A Coincidental Relationship?
}

\author{
Danielle Lee ${ }^{1}$, Bishoy Kolta ${ }^{2}$ and Karim Sedky ${ }^{3 *}$ \\ ${ }^{1}$ Second Year Medical Student, Cooper Medical School of Rowan University, USA \\ ${ }^{2}$ Third Year Psychiatry Resident, Cooper Medical School of Rowan University, USA \\ ${ }^{3}$ Psychiatry Medical Student Clerkship Director and Associate Professor of Psychiatry, Cooper Medical School of Rowan \\ University, USA
}

*Corresponding author: Karim Sedky, Psychiatry Medical Student Clerkship Director and Associate Professor, Department of Psychiatry, Cooper Medical School of Rowan University, 401 Haddon Ave, Suite 346 E and R building, Camden, NJ 08103, USA, Tel: 856-757-7799, Fax: 856-757-9651, E-mail: sedky66@hotmail.com

\begin{abstract}
A relationship between Polycystic Ovarian Syndrome (PCOS) and narcolepsy has not been well examined. Both disorders share common comorbidities including obesity, Obstructive Sleep Apnea (OSA), and excessive daytime sleepiness (EDS). However, it is not clear if these two disorders sporadically coexist or are commonly occurring and missed. Low or deficient hypocretin level as well as other hormonal abnormalities might explain this association. Authors present one case presentation supported by three other published cases and literature review about this comorbidity.
\end{abstract}

\section{Keywords}

Polycystic ovarian syndrome, Obstructive sleep apnea, Hypocretin, Narcolepsy, Cataplexy

\section{Introduction}

Narcolepsy is a disorder that affects sleep-wake cycles, causing individuals to experience periods of excessive daytime sleepiness (EDS), and sleep attacks. Individuals presenting with a sudden loss of muscle tone associated with strong emotions are diagnosed with narcolepsy with cataplexy. Other symptoms include sleep paralysis, hypnagogic and/or hypnopompic hallucinations, and disrupted nighttime sleep. Some individuals with narcolepsy have associated low or deficient hypocretin, a brain peptide that helps maintain alertness and regulates rapid eye movement sleep cycles. In the international classification of sleep disorders - third edition [1], two types of narcolepsy exists: type 1 is di- agnosed in the presence of cataplexy and either mean sleep latency test (MSLT) characteristics (i.e., short mean sleep latency $<8$ minutes and two or more sleep onset REM [SOREMs]; however one SOREM criteria can be replaced by REM occurring in the first 15 minutes of Polysomnography [PSG]) or Cerebrospinal Fluid (CSF) hypocretin deficiency (levels below $110 \mathrm{pg} / \mathrm{ml}$ or $<1 / 3$ of means of normal subjects). This is in contrast to type 2 which lacks cataplexy but has MSLT narcolepsy criteria and either normal or undocumented hypocretin levels.

Other causes of hypersomnia should be ruled out (i.e., medication or other substances as well as OSA or sleep phase disorders). Low hypocretin is thought to occur in high incidence in type 1 compared to type 2 . In one study [2] recruiting 26 narcoleptic patients with cataplexy and 9 narcoleptic patients without cataplexy, 23 individuals (88.5\%) with narcolepsy-cataplexy had low CSF hypocretin levels, compared to only one in the non-cataleptic group (11.11\%) had a low hypocretin level.

PCOS is one of the most common endocrinopathies diagnosed by Rotterdam criteria requiring at least two of these three features: 1) Oligo- or anovulation, 2) Clinical and/or biochemical signs of hyperandrogenism, and/or 3) Polycystic ovaries by ultrasound. EDS has been commonly reported in up-to $80 \%$ of this population, irrespective of their Body Mass Index (BMI) [3]. PCOS may occur at a young age in girls who develop ear-

Citation: Lee D, Kolta B, Sedky K (2017) Polycystic Ovarian Syndrome and Narcolepsy: A Coincidental Relationship?. J Sleep Disord Manag 3:015. doi.org/10.23937/2572-4053.1510015 Received: April 13, 2017: Accepted: June 26, 2017: Published: June 28, 2017

Copyright: (C) 2017 Lee D, et al. This is an open-access article distributed under the terms of the Creative Commons Attribution License, which permits unrestricted use, distribution, and reproduction in any medium, provided the original author and source are credited. 
ly pubarche and thelarche [4]. Researchers found a lower serum hypocretin in 36 women with PCOS compared to the control group [5]. In narcoleptic individuals, CSF hypocretin level lower than $110 \mathrm{pg} / \mathrm{ml}$ is included in the diagnostic criteria for narcolepsy. However, there is no simple equivalence between serum and CSF hypocretin level and only the latter is reliable for diagnosis of narcolepsy type 1.

In a study conducted by Tasali, et al. non-diabetic PCOS individuals were found to have poor sleep quality, daytime sleepiness, and increased risk for OSA, with higher fasting insulin levels in those at risk for OSA, independent of their obesity levels [6]. Additionally, extremely obese women with PCOS had significantly lower sleep efficiency, longer sleep-onset latency (SL), and less REM-sleep in comparison to healthy non-obese controls [6]. Although de Sousa found no differences in apnea-hypopnea index and other respiratory parameters among obese and extremely obese adolescents with PCOS versus controls with different body habitus, the above mentioned sleep pattern changes were noticed [7].

Both narcolepsy and PCOS thus share common symptoms including sleep disturbance, obesity and EDS. The purpose of this paper is to discuss published cases and discuss possible etiological factors comorbid for the two disorders.

\section{Case Report and Literature Review}

A 16-year-old hispanic female was diagnosed with PCOS who met two of Rotterdam criteria: she developed her puberty at age 9 with irregular menses persisting until age of 16; thus, treated with daily I-norgest and e-estradiol. Increased facial, hands and back hair were noticeable secondary to elevated testosterone $92 \mathrm{ng} / \mathrm{dl}$ (normal $=5-38 \mathrm{ng} / \mathrm{dl}$ ), decreased 17-hydroxypregnenolone 29 ( $n=44-235)$. In addition, her fasting insulin levels were elevated $34.7 \mathrm{IU} / \mathrm{ml}$ (normal $=2.6-24.9$ ) as well as $\mathrm{HbA} 1 \mathrm{C}$ was 5.9 ; thus, was medicated by metformin $500 \mathrm{mg}$ twice daily.

Associated hypothyroidism was detected necessitating levothyroxine $25 \mathrm{mg}$ daily (Thyroid Stimulating Hormone [TSH] was 1.97 (normal = 0.45-4.5). Other medical conditions included low blood pressure and stomach problems treated with omeprazole $40 \mathrm{mg}$ prior to each meal. Her BMI $=32.6 \mathrm{~kg} / \mathrm{m}^{2}$ and she had depressive symptoms. Her other routine blood work (including antistreptolysin O) and abdominal ultrasound over the ovaries which were normal.

Although she slept nine hours on daily basis, her initial complaint of EDS (Epworth Sleepiness Scale [ESS] = 17) was noticed at age 13 followed by sleep paralysis episodes about two years after. Eye lid dropping was noticed when she cried, developing three month after sleep paralysis onset. She slept at $10 \mathrm{pm}$ with SL of 30 minutes but would wake up 2-3 times. She woke up with difficulty at $7 \mathrm{am}$. Eight months prior to be seen, she started having six episodes of sleep paralysis which were frightening to her. She described her cataleptic attacks, which started three months after the sleep paralysis, as episodes of losing her muscle tone and eye drooping when she cries. She also reported sleep attacks that occurred during the day at school. Her PSG did not show respiratory events, but her sleep latency was 3 minutes and REM latency of 57 minutes. Additionally, her 5 nap MSLT showed sleep latency of 4.5 minutes and two SOREM. Her HLA DQB1 ${ }^{*} 0602$ and HLA DR15 were negative. Sleep log and actigraphy were never performed; however, according to the patient, her sleep pattern was consistent according to her report. After completing the diagnostic PSG/MSLT study, Armodafinil $50 \mathrm{mg}$ twice daily was prescribed which improved her symptoms but caused severe headache. This medication was discontinued and long-acting mixed amphetamine salts on a dose of $10 \mathrm{mg}$ daily was initiated with good tolerance and response. Patient was counseled on dieting and regular exercise as well as referral to a dietician. However, her compliance was poor with both.

Other cases have also been reported in the literature associating narcolepsy with patients having PCOS (Table 1). A 10-year-old female diagnosed with PCOS based on her amenorrhea complaints (she had signs of precocious puberty according to authors) and ultrasound showing polycystic ovaries. Her blood work showed hyperandrogenism and hyperinsulinaemia. She also was diagnosed with arachnoid cyst, and presented with EDS and cataplexy [8]. Due to her EDS, cataplexy, positive MSLT (PSG was normal, but her MSLT showed short SL of 1 minute and 4 SOREMs) and deficient CSF hypocretin levels, narcolepsy with cataplexy was diagnosed.

In yet another 25-year-old female diagnosed with PCOS, a presentation of obesity $\left(B M I=40.9 \mathrm{~kg} / \mathrm{m}^{2}\right)$, EDS (ESS = 19), OSA (Apnea-Hypopnea Index [AHI] = 15.4), was later found to also have narcolepsy. The diagnosis was confirmed with 4 SOREMs and short sleep latency between 0 to 2 minutes on MSLT. Symptoms improved with continuous positive pressure ventilation and the addition of modafinil. This later stimulant medication is commonly prescribed for managing narcolepsy symptoms [9]. Her PCOS was diagnosed based on the presence of oligomenorrhea approximately one menstruation a year since her menarche at age 18 , infertility indicating oligo or an-ovulation, obesity, hirsutism, elevated testosterone $(134.2 \mathrm{ng} / \mathrm{dl})$ and increased insulin of 489 $\mathrm{mU} / \mathrm{L}$ and C-peptide protein $>14$.

Another vaguely described case of a 14-year-old, overweight female, diagnosed with insulin resistance and PCOS, she presented with EDS, cataplexy, hallucinations and sleep paralysis confirming the diagnosis of narcolepsy [10]. She did not have sleep apnea but her MSLT was also normal (mean SL time was 11.2 minutes, 


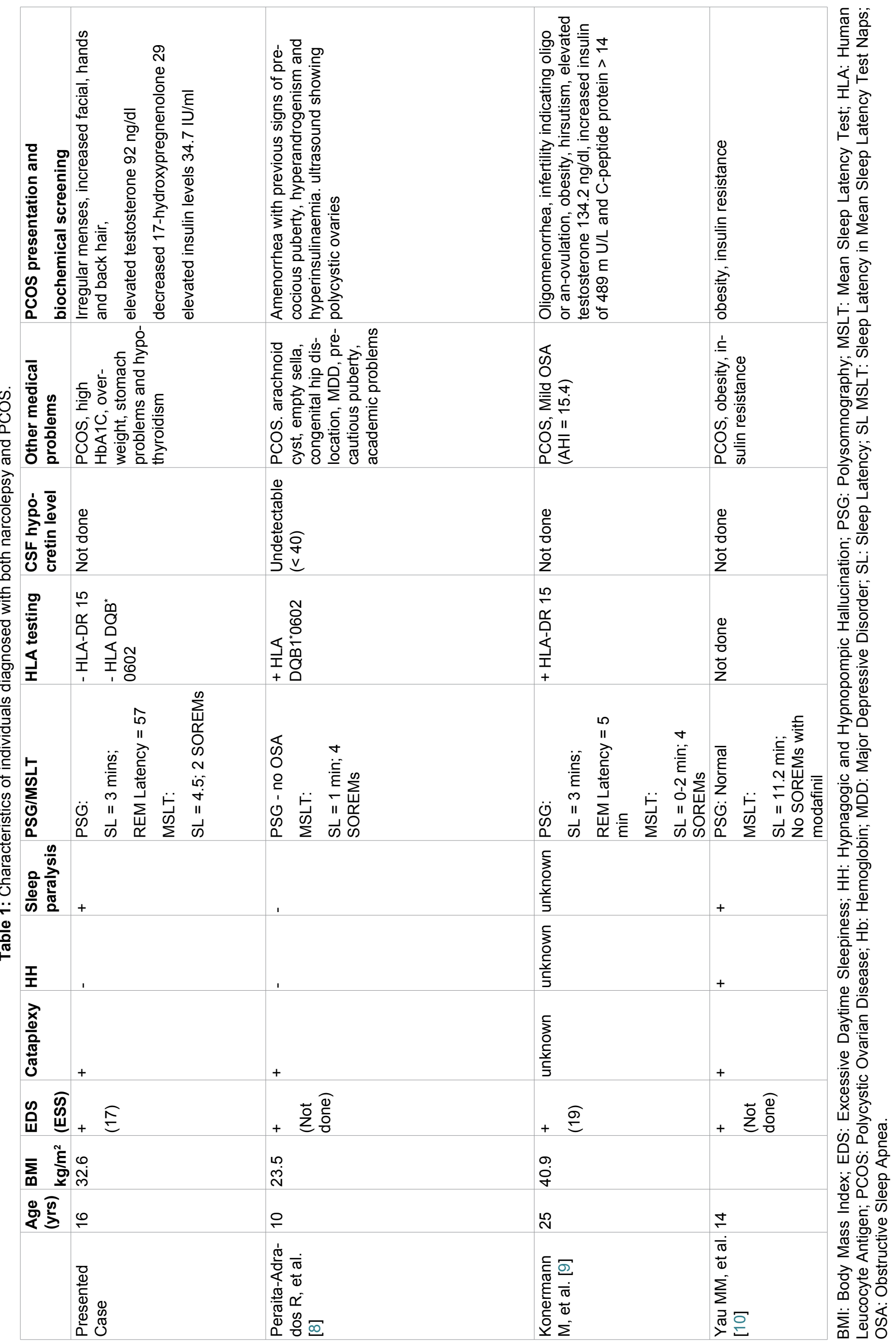


with no SOREMs). HLA typing and hypocretin-1 CSF level were not performed.

\section{Discussion}

Both Narcolepsy and PCOS present in the early adolescent period. Significant obesity has been reported, leading to increased risk for OSA. Obesity in Narcolepsy can be attributed to low activity and sedentary lifestyle. It can also be directly linked to hypocretin level which has been found to stimulate food intake. It also can counteract the effect of the satiety hormone Leptin. However, hypocretin also stimulates the release of Corticotropin Releasing Factor (CRF) which has been known to decrease food intake. One study showed that both CRF and Neuro Peptide Y (NPY) are involved in the effect of hypocretin on the food intake in rats suggesting that hypocretin effect on the food intake may be complex because of hypocretin-CRF and hypocretin-NPY linkage [11]. Low or deficient CSF hypocretin-1 can be found in majority of cases of narcolepsy with cataplexy, with lesser incidence in those without cataplexy. In obese individuals including PCOS, leptin resistance and decrease in ghrelin have been associated with hypocretin levels leading to EDS.

Although all narcoleptic individuals will have EDS, not all in the PCOS group will complain of this, irrespective of the OSA status or obesity. In a study, metformin supplementation to individuals diagnosed with PCOS decreased ESS compared to untreated group, although continued to be in the abnormal range, suggesting insulin resistance as a contributing factor for EDS [12]. Visceral fat and cytokines (interleukin- 6 and tumor necrosis factor $\alpha$ ) has been attributed to EDS as well. These presented cases had typical subjective and/or polysomnographic (with MSLT) features of narcolepsy, with this latter usually not examined in PCOS individuals.

Several hypothesis of the correlation between narcolepsy and PCOS can be considered. PCOS have been associated with low serum hypocretin levels [5] which may affect CSF hypocretin levels resulting in the full phenotype of narcolepsy and cataplexy. Serum and CSF hypocretin levels may linearly correlate in the same way serum and CSF glucose levels relate to each other. For example, the normal level of CSF glucose should be approximately $60 \%$ of the level of serum glucose [13].

PCOS could also cause sleepiness either via inflammatory or hormonal mechanism and it could also affect REM sleep. The combination of sleepiness and REM dyscontrol then yield a diagnosis of narcolepsy type- 2 in a hypocretin-independent mechanism. Since both types of narcolepsy are associated with obesity, which can be severe, the metabolic-hormonal dysregulation may thus be a manifestation of PCOS. Insulin resistance and hyperinsulinemia can occur in both normal-weight and overweight women with PCOS. Tasali, et al. showed that slow-wave sleep was specifically interrupted in humans with type-2 diabetes, and the magnitude of that interruption was dose correlated with the magnitude of insulin resistance [14].

In conclusion, correlation between PCOS and narcolepsy can be complex and multi-factorial. Although this publication discusses possible mechanisms that associate the two disorders, more studies are needed to assess the prevalence and co-occurrence of these two disorders and their possible association.

\section{Conflict of Interests}

Danielle Lee, Bishoy kolta and Karim sedky have no conflicts of interest.

\section{Authorship Contribution}

Danielle Lee and Karim Sedky evaluated and treated the patient. Both engaged in the initial literature search and drafting the manuscript. Bishoy Kolta expanded the literature search and finally edited the manuscript. Karim Sedky was responsible for the critical revision and final approval of the article.

\section{Acknowledgment}

None.

\section{References}

1. Ruoff C, Rye D (2016) The ICSD-3 and DSM-5 guidelines for diagnosing narcolepsy: clinical relevance and practicality. Curr Med Res Opin 20: 1-12.

2. Dauvilliers $Y$, Baumann $C R$, Carlander B, Bischof $M$, Blatter T, et al. (2003) CSF hypocretin-1 levels in narcolepsy, KleineLevin syndrome, and other hypersomnias and neurological conditions. J Neurol Neurosurg Psychiatry 74: 1667-1673.

3. Vgontzas AN, Legro RS, Bixler EO, Grayev A, Kales A, et al. (2001) Polycystic ovary syndrome is associated with obstructive sleep apnea and daytime sleepiness: role of insulin resistance. J Clin Endocrinol Metab 86: 517-520.

4. Bronstein J, Tawdekar S, Liu Y, Pawelczak M, David R, et al. (2011) Age of onset of polycystic ovarian syndrome in girls may be earlier than previously thought. J Pediatr Adolesc Gynecol 24: 15-20.

5. Yilmaz E, Celik O, Celik N, Simsek Y, Celik E, et al. (2013) Serum orexin-A (OXA) level decreases in polycystic ovarian syndrome. Gynecol Endocrinol 29: 388-390.

6. Tasali E, Van Cauter E, Ehrmann DA (2006) Relationships between sleep disordered breathing and glucose metabolism in polycystic ovary syndrome. J Clin Endocrinol Metab 91: 36-42.

7. de Sousa G, Schlüter B, Buschatz D, Menke T, Trowitzsch $E$, et al. (2010) A comparison of polysomnographic variables between obese adolescents with polycystic ovarian syndrome and healthy, normal-weight and obese adolescents. Sleep Breath 14: 33-38.

8. Peraita-Adrados R, García-Peñas JJ, Ruiz-Falcó L, Gutiérrez-Solana L, López-Esteban P, et al. (2011) Clinical, polysomnographic and laboratory characteristics of narcolepsy-cataplexy in a sample of children and adolescents. Sleep Med 12: 24-27.

9. Konermann M, Peine S, Rehling H, Rawert B (2003) PCO Syndrome and Sleep-related Breathing Disorders A Case Study. Sleep Research Online 5: 7-11. 
10. Yau MM, de Sousa C (2011) Paediatric Narcolepsy: A Rare and Easily Forgotten Diagnosis. HK J Paediatr 16: 131-138.

11. Ida T, Nakahara K, Kuroiwa T, Fukui K, Nakazato M, et al (2000) Both corticotropin releasing factor and neuropeptide $Y$ are involved in the effect of orexin (hypocretin) on the food intake in rats. Neurosci Lett 293: 119-122.

12. El-Sharkawy AA, Abdelmotaleb GS, Aly MK, Kabel AM (2014) Effect of metformin on sleep disorders in adolescent girls with polycystic ovarian syndrome. J Pediatr Adolesc Gynecol 27: 347-352.
13. Nigrovic LE, Kimia AA, Shah SS, Neuman MI (2012) Relationship between cerebrospinal fluid glucose and serum glucose. N Engl J Med 366: 576-578.

14. Tasali E, Leproult R, Ehrmann DA, Van Cauter E (2008) Slow-wave sleep and the risk of type 2 diabetes in humans. Proceedings of the National Academy of Sciences of the United States of America 105: 1044-1049. 